\title{
Extending the Graviton Propagator with a Lorentz-Violating Vector Field
}

\author{
Michael D. Seifert \\ Department of Physics, Astronomy, 8 Geophysics, Connecticut College \\ 270 Mohegan Ave., New London, CT 06375 \\ E-mail: mseifer1@conncoll.edu
}

\begin{abstract}
I discuss progress towards "bootstrapping" a Lorentz-violating gravity theory: namely, extending a linear Lorentz-violating theory of a rank-2 tensor to a non-linear theory by coupling this field to its own stress-energy tensor.
\end{abstract}

The gravitational sector of the Standard Model Extension (SME) has become of great interest in recent years, particularly with the recent detection of gravitational waves by the LIGO Collaboration. The treatment of gravity by the SME differs in an important way from its treatment of other sectors. In the context of a gravitationally curved spacetime, Lorentz violation cannot be thought of as due to a fixed background tensor; instead, the Lorentz-violating tensor field (represented abstractly by $\Psi^{\cdots \cdots}$ ) must have its own dynamics. ${ }^{1}$ The gravitational sector for the SME must therefore be thought of as including the Einstein-Hilbert action, various dynamical terms for $\Psi \cdots$, and small coupling terms between $\Psi \cdots$ and the Riemann tensor.

The great majority of the work thus far in the gravitational sector of the $\mathrm{SME}^{2-4}$ has focused on linearized perturbations about a solution where spacetime is flat $\left(g_{a b}=\eta_{a b}\right)$ and the Lorentz-violating tensor field is constant $(\nabla \Psi \cdots=0)$. In this limit, the dynamics of $\Psi \cdots$ do not greatly affect the gravitational dynamics. ${ }^{2}$ However, in strongly curved spacetimes, a constant tensor field $\Psi \cdots$ will in general not exist. If the SME framework is to address such spacetimes (such as compact objects, black holes, or cosmological spacetimes), we will have to address the dynamics of the underlying tensor field $\Psi^{\cdots}$.

An old idea in the context of gravitational physics is the idea of "bootstrapping" gravity from a linear theory to a non-linear theory. ${ }^{5}$ This method is based on the idea that "gravity gravitates": the stress-energy of the gravitational field should act as a source for the gravitational field. This idea 
predates the SME framework by a few decades, and it is instructive to ask whether one can extend this idea to situations with violation of Lorentz symmetry. In this process, three main questions arise:

(1) At the level of a linear free-field gravity theory, what kinds of theories can I write down if I allow for violations of Lorentz symmetry?

(2) Can such linear theories be bootstrapped to non-linear theories?

(3) Does requiring that the linear theory be "bootstrappable" place constraints on the dynamics of the Lorentz-violating field $\Psi \cdots$ ?

Let us consider a general linear theory of a source-free symmetric ranktwo tensor field $h_{a b}$ in flat spacetime, with action

$$
S=\frac{1}{2} \int d^{4} x \partial_{a} h_{b c} \mathcal{P}^{a b c d e f} \partial_{d} h_{e f}
$$

where the propagator tensor $\mathcal{P}^{\text {abcdef }}$ is some constant tensor to be determined. The resulting equations of motion are then

$$
\left(\mathcal{P}^{a b c d e f}+\mathcal{P}^{a e f d b c}\right) \partial_{a} \partial_{d} h_{e f}=0 .
$$

By symmetry in (1), we can assume that $\mathcal{P}^{a b c d e f}$ is symmetric under the exchanges $b \leftrightarrow c, e \leftrightarrow f$, and $\{a b c\} \leftrightarrow\{d e f\}$. From Eq. (2), we can also take the propagator to be symmetric under the exchange $\{b c\} \leftrightarrow\{e f\}$. Finally, we will want to insert a conserved stress-energy tensor as a source on the right-hand side of (2); this implies that the divergence of the lefthand side of (2) must also vanish. In Fourier space, this implies that the quantity $\mathcal{P}^{a b c d e f} k_{a} k_{b} k_{d}=0$ for all choices of wave propagation vector $k_{a}$.

We expect that in the end, $\mathcal{P}^{a b c d e f}$ will not be a fundamental object but rather a function of simpler tensors, such as a "fiducial" flat metric $\eta^{a b}$ or a Lorentz-violating tensor field of some kind. The strategy is then to write down the most general $\mathcal{P}^{a b c d e f}$ that can be constructed out of these simpler tensors, subject to the above symmetry constraints. Using the fiducial metric alone, for example, we find that the unique propagator satisfying the desired symmetry properties is the usual Lorentz-invariant linearized gravity propagator, as expected:

$$
\begin{aligned}
\left(\mathcal{P}_{L I}\right)^{a b c d e f}=\eta^{a(b} \eta^{c) d} \eta^{e f}+\eta^{a(e} \eta^{f) d} \eta^{b c}-\eta^{a(b} \eta^{c)(e} \eta^{f) d} & \\
& -\eta^{a(e} \eta^{f)(b} \eta^{c) d}-\eta^{a d} \eta^{b c} \eta^{e f}+\eta^{a d} \eta^{b(e} \eta^{f) c} .
\end{aligned}
$$

The simplest possible Lorentz-violating tensor field $\Psi^{\cdots}$ would be a four-vector field $A^{a}$. If we follow the above procedure, constructing the propagator out of $\eta^{a b}$ and $A^{a}$, we find that the resulting Lorentz-violating 
propagator $\left(\mathcal{P}_{L V}\right)^{\text {abcdef }}$ only has one free parameter $\xi$. What's more, this $\left(\mathcal{P}_{L V}\right)^{\text {abcdef }}$ is equivalent to the Lorentz-invariant $\left(\mathcal{P}_{L I}\right)^{\text {abcdef }}$ under the substitution

$$
\eta^{a b} \rightarrow \tilde{\eta}^{a b} \equiv \eta^{a b}+\xi A^{a} A^{b}
$$

In other words, the introduction of a Lorentz-violating vector field only allows one to change the "effective metric" $\tilde{\eta}^{a b}$ for linearized gravity. This will have the effect of changing the "light cones" for gravitational wave propagation; however, it will not allow for more exotic effects such as dispersion or birefringence of gravitational waves. This result is in agreement with the more general results of Ref. 4 .

Having classified the ways in which the linearized theory can break Lorentz symmetry, I now turn to the question of extending it to a nonlinear theory. To do this, I follow the method of Deser, ${ }^{6,7}$ and write down a first-order linear model in terms of a densitized tensor $\mathfrak{h}^{a b}$ and an auxiliary tensor $\Gamma_{b c}^{a}$. In the Lorentz-invariant case, the action for this model is

$$
S=\int d^{4} x\left[2 \mathfrak{h}^{a b} \partial_{[c} \Gamma_{b] a}^{c}+2 \eta^{a b} \Gamma_{d[c}^{c} \Gamma_{a] b}^{d}+\mathcal{L}_{\text {mat }}\left(\eta^{a b}, A^{a}, \partial_{a} A^{b}\right)\right] .
$$

The equations of motion for $\mathfrak{h}^{a b}$ and $\Gamma_{b c}^{a}$, combined, are equivalent to the linearized vacuum Einstein equations if we interpret $\mathfrak{h}^{a b}$ as the perturbation to the densitized inverse metric: $\mathfrak{g}^{a b}=\eta^{a b}+\mathfrak{h}^{a b}$. Under this interpretation, $\mathfrak{h}^{a b}$ must be coupled to the trace-reversed stress-energy $\tau_{a b}=\delta \mathcal{L} / \delta \eta^{a b}$. The second term in (5), along with the matter Lagrangian $\mathcal{L}_{\text {mat }}$, contribute to the stress-energy (note that the first term is independent of $\eta^{a b}$.) We will thus need to add two new coupling terms to the action (5):

$$
S \rightarrow S+\int d^{4} x \mathfrak{h}^{a b}\left[2 \Gamma_{d[c}^{c} \Gamma_{a] b}^{d}+\left(\tau_{\text {mat }}\right)_{a b}\right] .
$$

Importantly, the new term in the gravitational sector (the first term in (6)) does not depend on $\eta^{a b}$, and so the bootstrap procedure terminates here for the gravitational sector. In the matter sector, the term $\left(\tau_{\text {mat }}\right)_{a b}$ may itself depend on the metric $\eta^{a b}$, and so the contributions to the stress-energy from this coupling term must be added in as well. The iteration of this procedure can, in principle, generate an infinite series of terms. However, assuming that various integrability conditions are satisfied, ${ }^{7}$ the resulting series can be summed up to yield a matter action that is minimally coupled to the densitized metric $\mathfrak{g}^{a b}$. The gravitational terms, meanwhile, combine into the Palatini action for general relativity:

$$
S=\int d^{4} x\left[\mathfrak{g}^{a b} R_{a b}[\Gamma]+\mathcal{L}_{\text {mat }}\left(\mathfrak{g}^{a b}, A^{a}, \nabla_{a} A^{b}\right)\right]
$$


where the Ricci tensor $R_{a b}$ is viewed here as a function of the connection coefficients $\Gamma$.

Perhaps surprisingly, this scenario changes very little when we relax the assumption of Lorentz symmetry. As found above, the only possible modification that can be made to the linearized gravity action in the presence of a Lorentz-violating vector field is to replace the matter metric $\eta^{a b}$ with an effective metric $\tilde{\eta}^{a b}=\eta^{a b}+\xi A^{a} A^{b}$. This will give rise to a new term $\xi A^{a} A^{b} \Gamma_{d[c}^{c} \Gamma_{a] b}^{d}$ in the action; but this term is independent of $\eta^{a b}$, and so does not contribute to the stress-energy tensor. Thus, the entire bootstrap procedure carries through as before; the only difference is that the densitized metric that appears in the Palatini action is not the same as that appearing in the matter action:

$$
S=\int d^{4} x\left[\tilde{\mathfrak{g}}^{a b} R_{a b}[\Gamma]+\mathcal{L}_{\text {mat }}\left(\mathfrak{g}^{a b}, A^{a}, \nabla_{a} A^{b}\right)\right]
$$

where $\tilde{\mathfrak{g}}^{a b} \equiv \mathfrak{g}^{a b}+\xi A^{a} A^{b}$.

This action could then be rewritten using the (undensitized) gravitational metric as a fundamental variable; the result would be some kind of exotic tensor-vector theory of gravity. It is important to note, however, that the construction of this theory required that the action for the Lorentz-violating field $A^{a}$ itself be amenable to "bootstrapping"; in particular, it must satisfy various integrability constraints at each stage of the bootstrap procedure. I conjecture that a symmetry-breaking potential and a "Maxwell-type" kinetic term for $A^{a}$ will satisfy these constraints, and that more exotic kinetic terms will fail; but this has not yet been proven.

\section{Acknowledgments}

I would like to thank Connecticut College for their financial support in attending this conference.

\section{References}

1. V. A. Kostelecký, Phys. Rev. D 69, 105009 (2004).

2. Q. Bailey \& V. A. Kostelecký, Phys. Rev. D 74, 045001 (2006).

3. V. A. Kostelecký \& J. D. Tasson, Phys. Lett. B 749, 551-559 (2015).

4. V. A. Kostelecký \& M. Mewes, Phys. Lett. B 757, 510-514 (2016).

5. R. Kraichnan, Phys. Rev. 98, 1118-1122 (1955).

6. S. Deser, Gen. Rel. Grav. 1, 9-18 (1970).

7. V. A. Kostelecký \& R. Potting, Phys. Rev. D 79, 065018 (2009). 